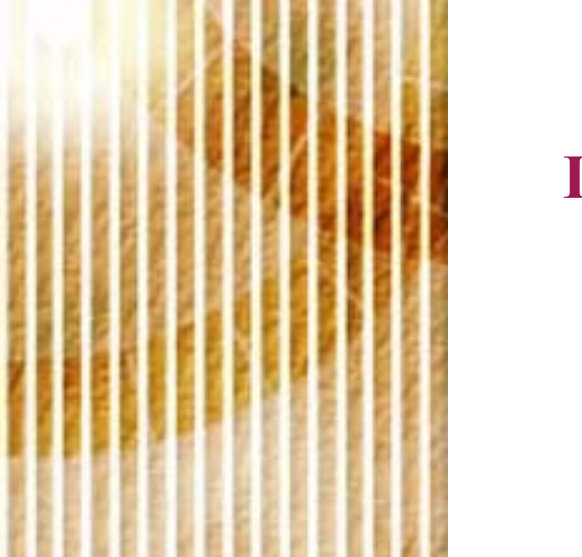

\title{
A Topology Optimization Method for Geometrically Nonlinear Problems Incorporating Level Set Boundary Expressions and a Particle Method*
}

\author{
Takayuki YAMADA**, Masatoshi MANABE**, Kazuhiro IZUI ${ }^{* *}$ and \\ Shinji NISHIWAKI** \\ ${ }^{* *}$ Graduate School of Engineering, Kyoto University \\ C3, Kyoto-daigaku-katsura, Nishikyo-ku, Kyoto, 615-8540, Japan \\ E-mail: takayuki@me.kyoto-u.ac.jp
}

\begin{abstract}
Structural topology optimization has been applied to nonlinear structural problems, but conventional methods considering geometrical nonlinearity encounter difficulties during nonlinear analysis using the FEM (Finite Element Method). In this study, we propose a new level set-based topology optimization method considering geometrical nonlinearity, using a meshfree particle technique, for optimizing elastic structures that undergo finite displacement. In the proposed method, the MPS (Moving Particle Semi-implicit) method is used for solving the state equation, since it does not use a mesh for geometrically nonlinear analysis. In this paper, first, a topology optimization problem is formulated based on the level set method, and a method for regularizing the optimization problem using the Tikhonov regularization method is explained. The reaction-diffusion equation that updates the level set function is then derived and an optimization algorithm, which uses the FEM to solve the reaction-diffusion equation when updating the level set function, is constructed. Next, the particle interaction model and the treatment of geometrical nonlinearity in the MPS method are described and the implementation that combines the level set-based topology optimization with the MPS method is explained. Finally, several numerical examples are provided to demonstrate the effectiveness of the proposed topology optimization method for geometrically nonlinear problems.
\end{abstract}

Key words : Topology Optimization, Particle Method, Level Set Method, Optimum Design, Structural Analysis, Geometrical Nonlinearity, Structural Optimization, Meshfree Method

\section{Introduction}

A primary feature of topology optimization ${ }^{(1)}$, the most flexible type of structural optimization, is replacement of the structural optimization problem by a material distribution problem in a fixed design domain. Topology optimization has been extensively applied to a variety of structural optimization problems, mainly dealing with linear materials that do not undergo large deformations.

Recently, however, topology optimization for structures has been applied to problems incorporating nonlinear materials and large deformations. To solve topology optimization problems for geometrically nonlinear structures, methods based on the homogenization design $\operatorname{method}^{(2),(3)}$, the density approach ${ }^{(4)-(11)}$, and the ECP (Element Connectivity Parameterization) method ${ }^{(12),(13)}$ have been proposed. Topology optimization of geometrically nonlinear structures can provide results that avoid unstable behavior, such as buckling, in response to design loads. 
Conventional topology optimization methods for structures with geometrical nonlinearity encounter difficulties during nonlinear analysis using the finite element (FE) method, due to the use of a mesh. In topology optimization using FE analysis based on the finite strain tensor for geometrically nonlinear problems, the tangent stiffness matrix becomes indefinite in low density finite elements, which prevents the convergence of solutions and renders such optimizations useless for geometrically nonlinear problems. This problem causes the appearance of grayscales in the optimal configurations of conventional topology optimizations, and distortion of finite elements during large deformation analysis. In prior research ${ }^{(4),(7)}$, to overcome these problems, nodes that constitute elements representing the weak material are removed from the tangent stiffness matrix in Newton-Raphson iterations. This procedure, however, complicates the FE analysis.

During the last decade, level set-based structural optimization methods were proposed (14)-(18) that are immune to the problem of grayscales. Level set-based topology optimization methods, such as Yamada's ${ }^{(19)-(21)}$, also enable topology optimization free of grayscales, theoretically, since shapes are expressed by the level set function, and such methods have been effectively applied to linear elastic problems. Certain shape optimization methods based on the level set method have been applied to geometrically nonlinear problems ${ }^{(16)-(18)}$, but few papers considering strong nonlinearity have been published. Furthermore, methods based on the Ersatz material approach ${ }^{(16),(19),(20)}$ are not perfectly free from grayscales numerically, since domains near the boundaries are represented as intermediate material.

The problem of distortion of the finite elements can be avoided by introducing a meshfree method, or a particle method, to replace the FEM. Meshfree methods can be classified into node-based methods that lack node and element connectivities, and element-based methods, so-called generalized FE methods, where mesh distortion is manageable. Various nodebased meshfree methods have been proposed, such as the EFGM (Element-Free Galerkin Method) ${ }^{(22)}$ and the RKPM (Reproducing Kernel Particle Method) ${ }^{(23),(24)}$. Concerning generalized FE methods, the X-FEM (eXtended Finite Element Method) ${ }^{(25),(26)}$ and others have been proposed. Generalized FE methods can be extended to the FEM to take advantage of developed techniques such as the use of different element types. However, it is difficult for meshfree methods to overcome tangent stiffness matrix problems that lead to calculation problems because, fundamentally, meshfree methods encounter the same difficulties as the FEM in this regard.

On the other hand, particle methods simulate a continuum as a set of particles. In particle methods, the deformation of the continuum is described by the deformation of the correlation of the particles. Particle methods that have been proposed include the SPH (Smoothed Particle Hydrodynamics) method ${ }^{(27)}$ and the MPS (Moving Particle Semi-implicit) method ${ }^{(28)}$ for fluid analysis, and both of these methods have recently been applied to nonlinear solid mechanics. In particular, the MPS method proposed by Koshizuka is extremely stable numerically and theoretically simple. The MPS method has been applied to elastostatic analysis ${ }^{(29),(30)}$, elastodynamic analysis ${ }^{(29),(30)}$, seismic response analysis, ${ }^{(31)}$ and elasto-plastic analysis ${ }^{(32)}$ in structural problems, and its efficiency has been demonstrated. Concerning topology optimization methods that use meshfree methods, a density approach using the $\mathrm{RKPM}^{(33)}$ and a topology optimization method based on an implicit topology description using the RKPM ${ }^{(34)}$ have been proposed. However, particle methods have not been applied to topology optimization problems, despite their effectiveness.

In this study, we propose a new level set-based topology optimization method incorporating a particle method to enable consideration of geometrical nonlinearity, for optimizing elastic structures that undergo large deformations. In the proposed method, the MPS method is used for the displacement response analysis since it does not rely on a finite element mesh for geometrically nonlinear analysis. The particles are deployed in the material domain represented by the level set function at every iteration. That is, the fixed design domain is perfectly free from grayscales and fictitious weak materials, since the Ersatz material approach is not 
used.

In Section 2, a topology optimization problem incorporating a level set boundary expression is formulated. In Section 3, an optimization algorithm is constructed based on the topology optimization problem and geometrically nonlinear analysis using the MPS method, and a strategy for integrating these two optimization components is described. In Section 4 , the effectiveness of the proposed optimization method is confirmed for a minimum end compliance problem using numerical examples.

\section{Formulations}

\subsection{Level Set-Based Topology Optimization}

Consider a structural optimization problem that is to determine the optimal configuration of a domain filled with a solid material, i.e., a material domain $\Omega$ that denotes the design domain, by minimizing an objective functional $F$ under a constraint functional $G$ operating as a volume constraint, described as follows:

$$
\begin{array}{ll}
\inf _{\Omega} & F[\Omega]=\int_{\Omega} f(\boldsymbol{x}) \mathrm{d} \Omega \\
\text { subject to } & G[\Omega]=\int_{\Omega} \mathrm{d} \Omega-V_{\max } \leq 0,
\end{array}
$$

where $V_{\max }$ is the upper limit of the volume constraint and $\boldsymbol{x}$ represents a point located in $\Omega$. In conventional topology optimization methods, a fixed design domain $D$, composed of a material domain $\Omega$ such that $\Omega \subset D$, and another complementary domain representing a void exist. That is, a void domain $D \backslash \Omega$ is introduced. Using the characteristic function $\chi_{\Omega} \in L^{\infty}$, defined as

$$
\chi_{\Omega}=\left\{\begin{array}{lll}
1 & \text { if } & x \in \Omega \\
0 & \text { if } & x \in D \backslash \Omega,
\end{array}\right.
$$

the above structural optimization problem is replaced by a material distribution problem, to search for an optimal configuration of the design domain in the fixed design domain $D$, as follows:

$$
\begin{array}{ll}
\inf _{\chi_{\Omega}} & F\left[\chi_{\Omega}(\boldsymbol{x})\right]=\int_{D} f(\boldsymbol{x}) \chi_{\Omega}(\boldsymbol{x}) \mathrm{d} \Omega \\
\text { subject to } & G\left[\chi_{\Omega}(\boldsymbol{x})\right]=\int_{D} \chi_{\Omega}(\boldsymbol{x}) \mathrm{d} \Omega-V_{\max } \leq 0 .
\end{array}
$$

Now, we briefly discuss the basic concept of a level set-based topology optimization method that includes the Tikhonov regularization method. We use the level set method to represent the structural boundaries, using a scalar function, the so-called level set function. As shown in the following equation, the level set function $\phi(x)$ assumes a positive value in the material domain, a negative value in the void domain, and a value of zero on structural boundaries.

$$
\begin{cases}0<\phi(x) \leq 1 & \text { for } \quad \forall x \in \Omega \backslash \partial \Omega \\ \phi(x)=0 & \text { for } \quad \forall x \in \partial \Omega \\ -1 \leq \phi(x)<0 & \text { for } \quad \forall x \in D \backslash \Omega .\end{cases}
$$

Based on the above structural boundary expression, the topology optimization problem is formulated as

$$
\begin{array}{lll}
\inf _{\phi} & F\left[\chi_{\phi}(\phi)\right]=\int_{D} f(\boldsymbol{x}) \chi_{\phi} \mathrm{d} \Omega \\
\text { subject to } & G\left[\chi_{\phi}(\phi)\right]=\int_{D} \chi_{\phi} \mathrm{d} \Omega-V_{\max } \leq 0,
\end{array}
$$

where $\chi_{\phi}(\phi)$ is a characteristic function defined as follows:

$$
\chi_{\phi}(\phi)= \begin{cases}1 & \phi \geq 0 \\ 0 & \phi<0 .\end{cases}
$$


Next, the above optimization problem is regularized. In conventional topology optimization methods, the homogenization method or a density approach is used for regularization, but the use of a level set boundary expression here prevents the use of these techniques. To overcome this problem, Yamada et al. proposed a new type of regularization technique based on the introduction of the Tikhonov regularization term into the objective functional ${ }^{(19),(20)}$. That is, the above topology optimization problem (7), (8), is replaced by the following:

$$
\begin{array}{ll}
\inf _{\phi} & F_{R}\left[\chi_{\phi}(\phi), \phi\right]=\int_{D} f(\boldsymbol{x}) \chi_{\phi} \mathrm{d} \Omega+\int_{D} \frac{1}{2} \tau|\nabla \phi|^{2} \mathrm{~d} \Omega \\
\text { subject to } & G\left[\chi_{\phi}(\phi)\right]=\int_{D} \chi_{\phi} \mathrm{d} \Omega-V_{\max } \leq 0,
\end{array}
$$

where $F_{R}$ is the regularized objective functional and $\tau>0$ is a regularization parameter.

In the proposed method, the existence of the gradient of the level set function is assumed. To regularize the topology optimization problem, the objective functional is replaced by the sum of the objective functional and the square of the gradient of the level set function. As shown in Fig. 1, the level set function has a value of -1 in the void domain and 1 in the material domain, and the level set function is distributed smoothly as a scalar function near the boundary where $\phi(x)=0$. Next, the optimization problem represented by (10) and (11)

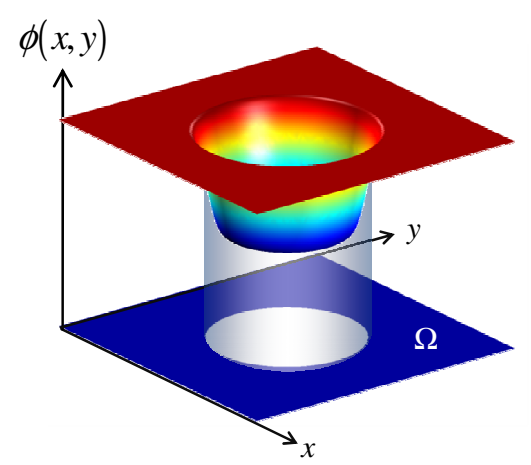

Fig. 1 Fixed design domain $D$ and level set function $\phi$

is reformulated using Lagrange's method of undetermined multipliers. Let the Lagrangian be $\overline{F_{R}}$ and the Lagrange multiplier of the volume constraint be $\lambda$. The optimization problem is then formulated as

$$
\inf _{\phi} \overline{F_{R}}\left[\chi_{\phi}(\phi), \phi\right]=F_{R}+\lambda G
$$

The optimal configuration will be obtained by solving the above optimization problem.

The necessary optimality conditions (KKT-conditions) for the above optimization problem are now derived, as follows:

$$
{\overline{F_{R}}}^{\prime}=0, \quad \lambda G=0, \quad \lambda \geq 0, \quad G \leq 0 .
$$

The level set function describing the optimal configurations satisfies the above KKT-conditions. Conversely, solutions obtained by Eq. (13) are optimum solution candidates, but obtaining this level set function directly is problematic, so the optimization problem is replaced by a problem of solving time evolutional equation that will provide optimum solution candidates.

\subsection{Time Evolutional Equation}

Here, we assume that variation of the level set function $\phi$ with respect to fictitious time $t$ is proportional to the gradient of the Lagrangian $\bar{F}$, as shown in the following:

$$
\frac{\partial \phi}{\partial t}=-K{\overline{F_{R}}}^{\prime}
$$


where $K>0$ is a coefficient of proportionality. Substituting Eq. (12) into Eq. (14) and then setting an appropriate boundary condition yields the following equation ${ }^{(19)}$ :

$$
\left\{\begin{array}{l}
\frac{\partial \phi}{\partial t}=-K\left(\bar{F}^{\prime}-\tau \nabla^{2} \phi\right) \\
\frac{\partial \phi}{\partial n}=0 \quad \text { on } \quad \partial D \backslash \partial D_{N} \\
\phi=1 \quad \text { on } \quad \partial D_{N},
\end{array}\right.
$$

where $\partial D_{N}$ is non-design boundaries. In this paper, optimum solution candidates are found by solving Eq. (15).

\subsection{Minimum End Compliance Problem}

The method proposed above is now applied to a minimum end compliance problem considering geometrical nonlinearity. In this method, the end compliance $F_{\text {e.c. }}$ is the objective function which is to be minimized. The end compliance represents the mean compliance at an end state, and is handled simply as an extension of the linear problem. Consider a material domain where the displacement is fixed at one boundary and a force is imposed at another boundary. A body force may also be applied throughout the material domain. The minimum end compliance problem under a volume constraint is then formulated as

$$
\begin{array}{ll}
\inf _{\phi} & F_{\text {e.c. }}=l(\boldsymbol{u}) \\
\text { subject to } & R(\boldsymbol{u}, \boldsymbol{v})=l(\boldsymbol{v})-a(\boldsymbol{u}, \boldsymbol{v})=0 \\
& \text { for } \forall \boldsymbol{v} \in U \boldsymbol{u} \in U \\
& G \leq 0,
\end{array}
$$

where the above elements are defined as

$$
\begin{aligned}
& a(\boldsymbol{u}, \boldsymbol{v})=\int_{D} \boldsymbol{\epsilon}(\boldsymbol{u}): \mathbf{E}: \boldsymbol{\epsilon}(\boldsymbol{v}) \chi_{\phi} \mathrm{d} \Omega \\
& l(\boldsymbol{v})=\int_{\Gamma_{t}} \boldsymbol{P}_{\text {ext }} \cdot \boldsymbol{v} \mathrm{d} \Gamma+\int_{D} \mathbf{b} \cdot \boldsymbol{v} \chi_{\phi} \mathrm{d} \Omega \\
& G=\int_{D} \chi_{\phi} \mathrm{d} \Omega-V_{\max },
\end{aligned}
$$

where $\boldsymbol{\epsilon}$ is the strain tensor defined in the MPS method, $\mathbf{E}$ is the elasticity tensor, $\boldsymbol{u}$ is the state, $v$ is the adjoint state, and

$$
U=\left\{\boldsymbol{v}=v_{i} \mathbf{e}_{i}: v_{i} \in H^{1}(D) \text { with } \boldsymbol{v}=0 \text { on } \Gamma_{u}\right\} .
$$

In the MPS method, the strain tensor $\epsilon$ is

$$
\boldsymbol{\epsilon}(\boldsymbol{u})=\sqrt{\mathbf{F}^{T} \mathbf{F}}-\mathbf{I},
$$

where $\mathbf{F}$ is the deformation gradient tensor and $\mathbf{I}$ is the identity tensor. Equation (23) represents the strain tensor independently of rigid rotation, and is the first-order formula for the deformation gradient tensor F. Details pertaining to Eq. (23) are given in 3.3.

Next, the sensitivity of regularized Lagrangian $\overline{F_{\text {e.c. }}}$ for the minimum compliance problem is derived. The regularized Lagrangian $\overline{F_{e . c .}}$ is the following:

$$
\overline{F_{e . c .}}=l(\boldsymbol{u})+R(\boldsymbol{u}, \boldsymbol{v})+\lambda G .
$$

Using Eq. (24), the KKT-conditions are derived as follows.

$$
{\overline{F_{\text {e.c. }}}}^{\prime}=0, \quad R(\boldsymbol{u}, \boldsymbol{v})=0 \quad \text { for } \boldsymbol{u}, \forall \boldsymbol{v} \in U, \quad \lambda G=0, \quad \lambda \geq 0, \quad G \leq 0
$$

The sensitivity of Lagrangian $\overline{F_{\text {e.c. }}}$ is obtained using the adjoint variable method as

$$
{\overline{F_{e . c .}}}^{\prime}=\left\langle\frac{\partial l(\boldsymbol{u})}{\partial \boldsymbol{u}}, \delta \boldsymbol{u}\right\rangle+\left\langle\frac{\partial R(\boldsymbol{u}, \boldsymbol{v})}{\partial \boldsymbol{u}}, \delta \boldsymbol{u}\right\rangle+\left\langle\frac{\partial R(\boldsymbol{u}, \boldsymbol{v})}{\partial \phi}, \delta \phi\right\rangle+\lambda\left\langle\frac{\partial G}{\partial \phi}, \delta \phi\right\rangle
$$


for $\forall \delta \boldsymbol{u} \in U$.

To eliminate the sensitivity with respect to $\frac{\mathrm{d} u}{\mathrm{~d} \phi}$, the adjoint variable field $v$ is chosen such that

$$
\left\langle\frac{\partial l(\boldsymbol{u})}{\partial \boldsymbol{u}}, \delta \boldsymbol{u}\right\rangle+\left\langle\frac{\partial R(\boldsymbol{u}, \boldsymbol{v})}{\partial \boldsymbol{u}}, \delta \boldsymbol{u}\right\rangle=0
$$

Therefore, finally, the sensitivity of Lagrangian $\overline{F_{\text {e.c. }}}$ is defined as

$$
{\overline{F_{e . c .}}}^{\prime}=\left\langle\frac{\partial}{\partial \phi}(R(\boldsymbol{u}, \boldsymbol{v})+\lambda G), \delta \phi\right\rangle .
$$

\section{Numerical implementations}

\subsection{Optimization Algorithm}

The flowchart of the optimization procedure is shown in Fig. 2. As shown, the initial configuration is set first. In the second step, the equilibrium equations are solved using the MPS method and the objective functional is computed in the third step. The optimization process finishes if the objective functional has converged, otherwise the sensitivities with respect to the objective functional are computed and the level set function $\phi$ is updated based on Eq. (15), using the finite element method.

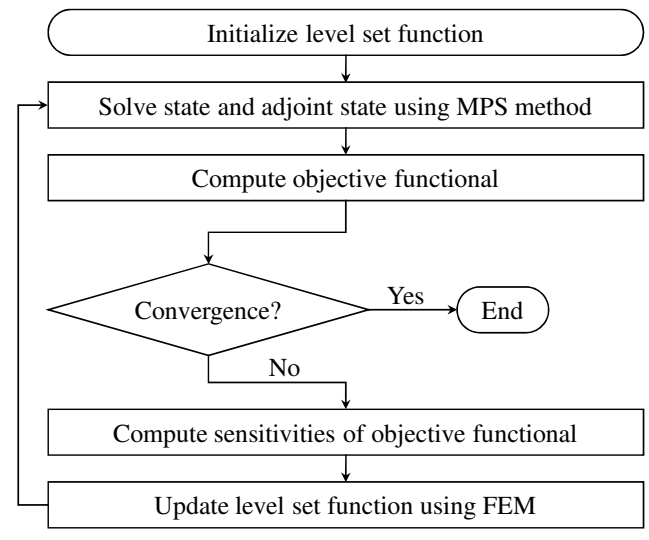

Fig. 2 Flowchart of the optimization procedure

\subsection{The MPS Method}

The MPS method is a discretization method for continua using interactive particles and a model based on differential operators. In the MPS method, a weighting function $w$ is defined as

$$
w\left(\left|\mathbf{r}_{i j}^{0}\right|\right)= \begin{cases}\frac{r_{e}}{\left|\mathbf{r}_{i j}^{0}\right|}-1 & \left(0 \leq\left|\mathbf{r}_{i j}^{0}\right|<r_{e}\right) \\ 0 & \left(r_{e} \leq\left|\mathbf{r}_{i j}^{0}\right|\right),\end{cases}
$$

where $\mathbf{r}_{i j}^{0}$ is the initial relative distance vector between particles $i$ and $j$, and $r_{e}$ is the effective radius of interaction of the particles, which here is set so that $r_{e}$ is $3.1 \times \mathbf{r}_{i j}^{0}$. The weighting function $w$ refers to the number of particles that each particle is connected to. The particle number density $n_{i}^{0}$ of particle $i$ is defined as the sum of weighting functions $w$ at the location of particle $i$, as follows.

$$
n_{i}^{0}=\sum_{j \in \text { Neighbor }} w\left(\left|\mathbf{r}_{i j}^{0}\right|\right)
$$


In the MPS method applied here, the governing equation is discretized as follows, using the gradient and divergence operators defined in the above models.

$$
\begin{aligned}
& \langle\nabla \phi\rangle_{i}=\frac{d}{n^{0}} \sum_{j \neq i}\left[\frac{\phi_{j}-\phi_{i}}{\left|\mathbf{r}_{j}-\mathbf{r}_{i}\right|}\left(\mathbf{r}_{j}-\mathbf{r}_{i}\right) w\left(\left|\mathbf{r}_{j}-\mathbf{r}_{i}\right|\right)\right] \\
& \langle\nabla \cdot \boldsymbol{u}\rangle_{i}=\frac{d}{n^{0}} \sum_{j \neq i} \frac{\left(\boldsymbol{u}_{j}-\boldsymbol{u}_{i}\right) \cdot\left(\mathbf{r}_{j}-\mathbf{r}_{i}\right)}{\left|\mathbf{r}_{j}-\mathbf{r}_{i}\right|^{2}} w\left(\left|\mathbf{r}_{j}-\mathbf{r}_{i}\right|\right)
\end{aligned}
$$

\subsection{Geometrical Nonlinearity in the MPS Method}

In the MPS method shown in Fig. 3, the relative displacement between particles $i$ and $j$ is defined as

$$
\boldsymbol{u}_{i j}=\mathbf{r}_{i j}-\mathbf{R} \mathbf{r}_{i j}^{0},
$$

where $\mathbf{r}_{i j}$ is the current relative distance vector between particles $i$ and $j$, and $\mathbf{R}$ is the orthogonal rotation tensor. Equation (33) can be rewritten as

$$
\boldsymbol{u}_{i j}=\mathbf{F r}_{i j}^{0}-\mathbf{R r}_{i j}^{0},
$$

so that the strain tensor in the MPS method is defined as

$$
\boldsymbol{\epsilon}=\mathbf{F}-\mathbf{R},
$$

where deformation gradient tensor $\mathbf{F}$ is the following:

$$
\mathbf{F} \simeq \boldsymbol{u}+\mathbf{R}-\mathbf{I}
$$

where $\boldsymbol{u}$ is the right stretch tensor. Substituting Eq. (36) into Eq. (35),

$$
\boldsymbol{\epsilon}=\boldsymbol{u}-\mathbf{I}
$$

where $\boldsymbol{u}^{2}=\boldsymbol{u} \cdot \boldsymbol{R}^{T} \cdot \boldsymbol{R} \cdot \boldsymbol{u}=\mathbf{F}^{T} \mathbf{F}=\mathbf{C}$ and $\mathbf{C}$ is the right Cauchy-Green tensor. Therefore, strain tensor $\boldsymbol{\epsilon}$ in Eq. (23) is derived. In other words, in the MPS method, the strain tensor $\boldsymbol{\epsilon}$ has the orthogonal tensor $\mathbf{R}$ removed from the deformation gradient tensor $\mathbf{F}$. The strain tensor $\boldsymbol{\epsilon}$ is therefore independent with respect to rigid rotation.

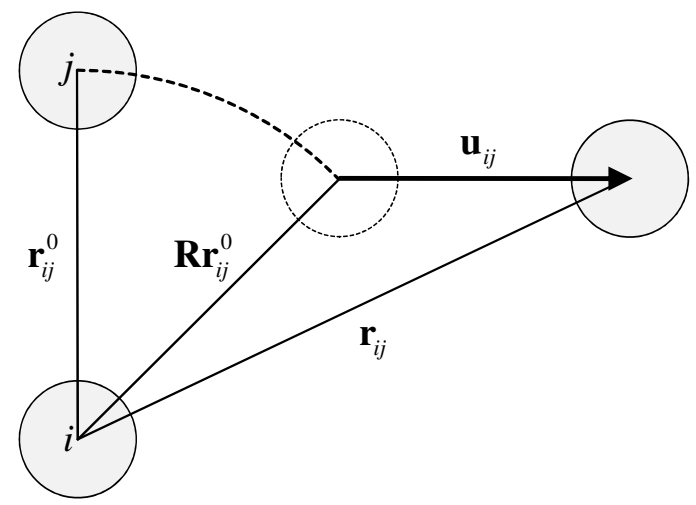

Fig. 3 Definition of relative displacement $\boldsymbol{u}_{i j}$ in the MPS method

\subsection{Application of the MPS Method in the Proposed Level Set-Based Topology Opti-} mization Method

We apply the MPS method to the proposed topology optimization method incorporating a level set boundary expression to handle the issue of large deformations. In the level setbased topology optimization procedure shown in Fig. 2, the end compliance as an objective functional is calculated by geometrically nonlinear analysis using the MPS method. 
In this study, the level set function is discretized using the finite element method, since the fixed design domain is not changed during the optimization process. In the proposed method, first, the particles are set on each finite element. In the second step, particles in the void domain $(\phi(x)<0)$ are removed to represent the void domain, as shown in Fig. 4. Therefore, the
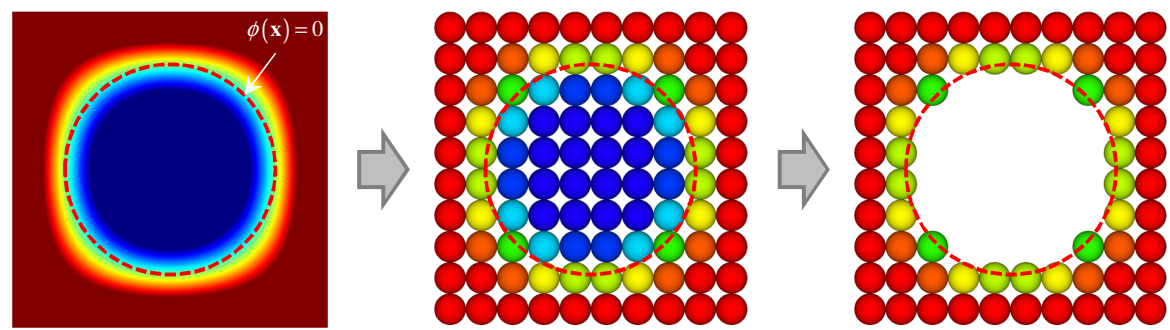

Fig. 4 Illustration of particles in the void domain being ignored in the MPS method

proposed method is perfectly free from grayscales for the intermediate domain and fictitious weak material for void domain. As a result, this scheme avoids numerical instabilities caused by geometrical nonlinearity ${ }^{(4),(7)}$.

\section{Numerical Examples}

\subsection{Topology Optimization of a Fixed Supported Beam under Single Loading}

In this section, two typical numerical examples are presented to confirm the effectiveness of the proposed optimization method for two-dimensional minimum end compliance problems. First, using a minimum end compliance problem considering geometrical nonlinearity for a fixed supported beam, the most typical numerical example in the literature, we examine the effects of different design loads, initial configurations, and particle sizes upon the resulting optimal configurations. Fig. 5 shows the fixed design domain and boundary conditions for the fixed supported beam design problem. In these examples, the isotropic elastic material has Young's modulus $=1 \mathrm{MPa}$ and Poisson's ratio $=0.3$. The upper limit of the volume constraint $V_{\max }$ is set to $20 \%$ of the volume of the fixed design domain.

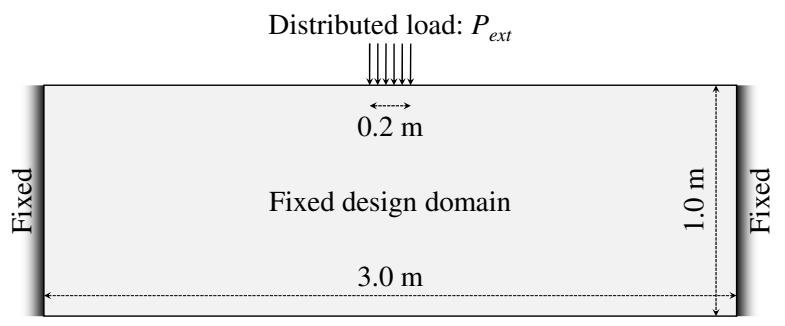

Fig. 5 Fixed design domain and boundary conditions for fixed supported beam design problem

\subsubsection{Effect of Design Loads}

First, we examine the effect of different design loads upon the resulting optimal configurations. In the level set function field, the fixed design domain is discretized using a structured mesh and four-node quadrilateral finite elements $1.0 \times 10^{-2} \mathrm{~m}$ in length. In the displacement field, the fixed design domain is discretized using particles $1.0 \times 10^{-2} \mathrm{~m}$ in size. Figure 6 shows the initial configuration. Figure 7(a) shows the optimal configuration using linear finite element analysis under a design load where $P_{\text {ext }}=1 \mathrm{~N} / \mathrm{m}^{2}$, and the result is a structure of two frames under compression only. Figure 7(b) shows the optimal configuration using linear finite element analysis under a design load where $P_{\text {ext }}=4,000 \mathrm{~N} / \mathrm{m}^{2}$. These results show that the optimal configurations are independent of the design loads when using linear finite element analysis. Figure 8(a) shows the optimal configuration using geometrically nonlinear 
analysis with the MPS method under a design load where $P_{\text {ext }}=1 \mathrm{~N} / \mathrm{m}^{2}$. The result appears to be identical with the result when linear finite element analysis is used because the design load $P_{\text {ext }}$ is very small. Figure 8 (b) shows the optimal configuration using geometrically nonlinear analysis with the MPS method under a design load where $P_{\text {ext }}=4,000 \mathrm{~N} / \mathrm{m}^{2}$. The result is an optimal structure that avoids buckling under the set design load. The nonlinear analysis results indicate that the proposed method obtains optimal configurations that are appropriate for the imposed design loads.

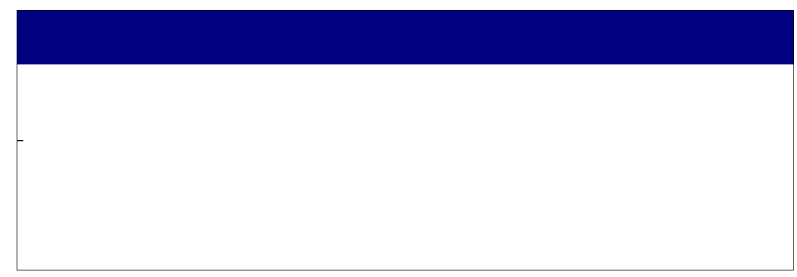

Fig. 6 Initial configuration for fixed supported beam

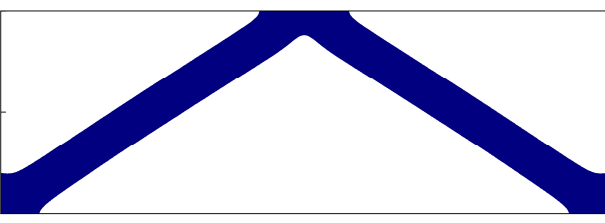

(a) $P_{e x t}=1 \mathrm{~N} / \mathrm{m}^{2}$

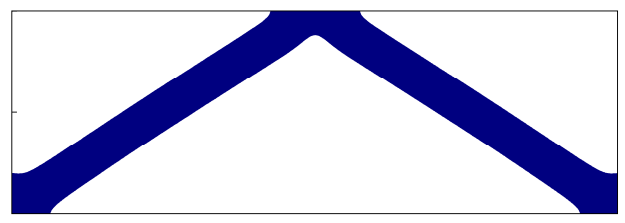

(b) $P_{e x t}=1,250 \mathrm{~N} / \mathrm{m}^{2}$

Fig. 7 Optimal configurations using the linear finite element method

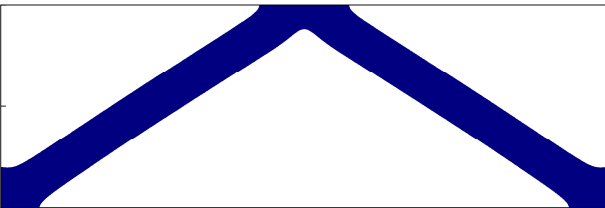

(a) $P_{e x t}=1 \mathrm{~N} / \mathrm{m}^{2}$

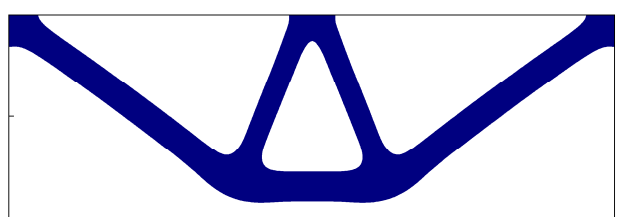

(b) $P_{\text {ext }}=1,250 \mathrm{~N} / \mathrm{m}^{2}$

Fig. 8 Optimal configurations using the MPS method

\subsubsection{Effect of Initial Configuration}

Second, we examine the effect of different initial configurations upon the resulting optimal configurations. In the level set function field, the fixed design domain is discretized using a structured mesh and four-node quadrilateral finite elements $1.0 \times 10^{-2} \mathrm{~m}$ in length. In the displacement field, the fixed design domain is discretized using particles $1.0 \times 10^{-2} \mathrm{~m}$ in size. The design load $P_{\text {ext }}$ is $4,000 \mathrm{~N} / \mathrm{m}^{2}$.

Figure 9 shows two cases and the obtained optimal configurations, each using a different initial configuration. The initial configuration for Case 1 has 27 holes; the initial configuration for Case 2 has 75 holes. In both cases, the optimal configurations are smooth, clear and nearly the same. That is, an appropriate optimal configuration was obtained for both initial configurations, confirming that the dependency of the obtained optimal configurations upon the initial configuration is extremely low.

\subsubsection{Effect of Particle Size}

Third, we examine the effect of the particle size upon the resulting optimal configurations, using the initial configuration shown in Fig. 6. We examine four cases whose degree 


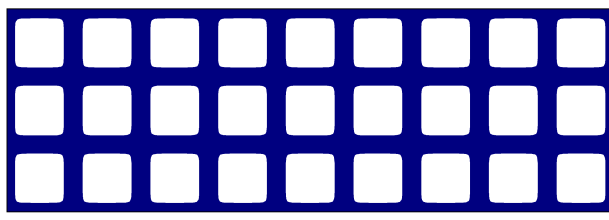

Initial configuration

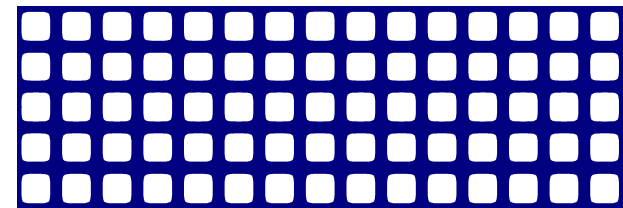

Initial configuration

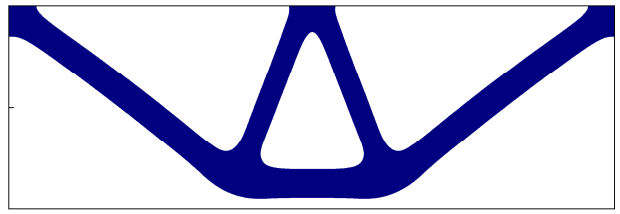

Optimal configuration

(a) Case 1

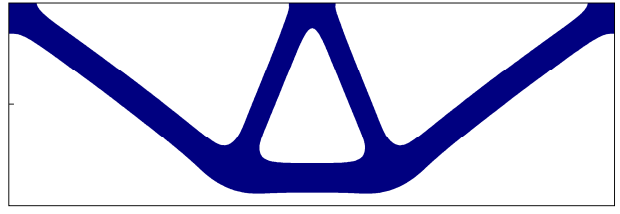

Optimal configuration

(b) Case 2

Fig. 9 Effect of initial configurations

of discretization is varied by using the following numbers of particles: $19,200(80 \times 240)$, $30,000(100 \times 300), 76,800(160 \times 480)$, and 120,000 $(200 \times 600)$.

Figure 10 shows the optimal configuration for each of the four cases. In all cases, the optimal configurations are smooth, clear, and nearly the same. That is, appropriate optimal configurations can be obtained despite a wide variation in the degree of discretization that was used. Thus, we can confirm that dependency with regard to the finite element mesh size of the level set function is extremely small, provided that the finite element size is sufficiently small.

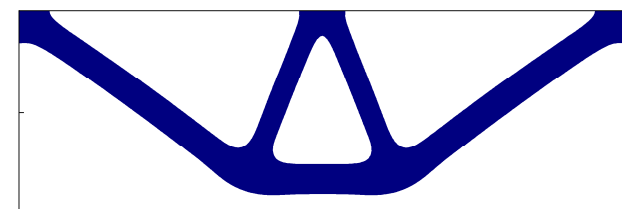

(a) $19200(80 \times 240)$ particles

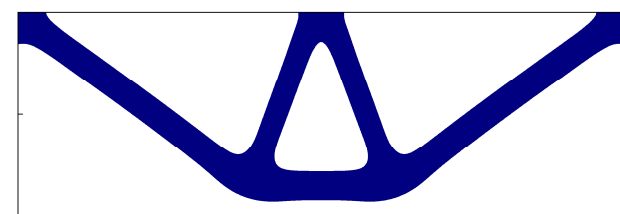

(c) $76800(160 \times 480)$ particles

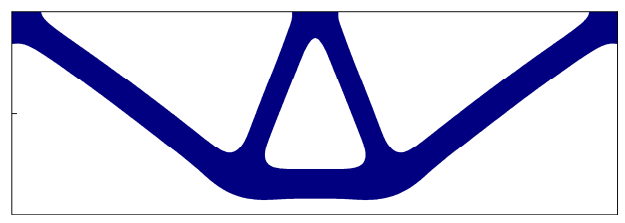

(b) $30000(100 \times 300)$ particles

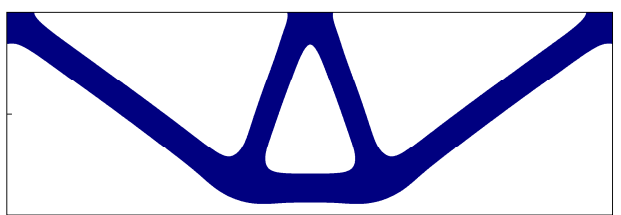

(d) $120000(200 \times 600)$ particles

Fig. 10 Effect of particle size

\subsection{Topology Optimization for a Simple Supported Beam}

Next, we apply the proposed method to the topology optimization of a simple supported beam structure. Figure 11 shows the fixed design domain and boundary conditions of this design problem. In the level set function field, the fixed design domain is discretized using a structured mesh and four-node quadrilateral finite elements $1.0 \times 10^{-2} \mathrm{~m}$ in length. In the displacement field, the fixed design domain is discretized using particles whose size is $1.0 \times$ $10^{-2} \mathrm{~m}$. In this example, the isotropic elastic material also has Young's modulus $=1 \mathrm{MPa}$ and Poisson's ratio $=0.3$, but the upper limit of the volume constraint $V_{\max }$ is set to $30 \%$ of the volume of the fixed design domain. Figure 12 shows the initial configuration.

Figures 13(a) and (b) show optimal configurations when linear finite element analysis is used. As demonstrated in Section 4.1.1, the optimal configurations are independent of the 


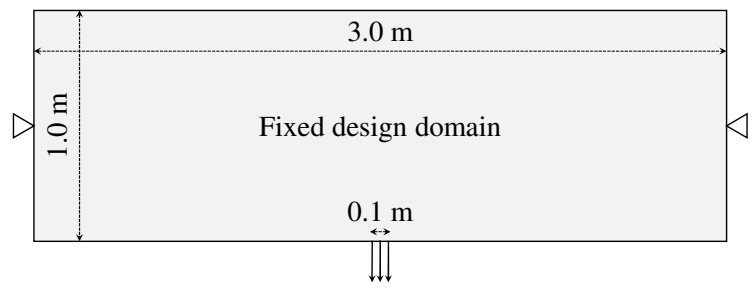

Distributed load: $P_{e x t}$

Fig. 11 Fixed design domain and boundary conditions of design problem for simple supported beam

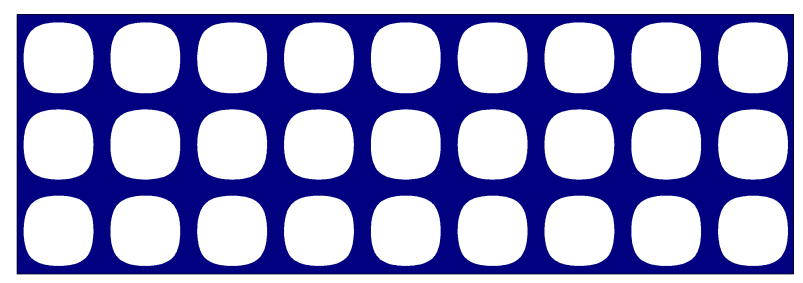

Fig. 12 Initial configuration for simple supported beam

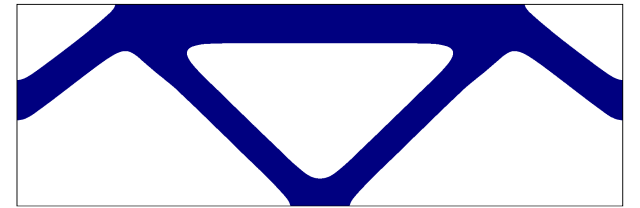

(a) $P_{\text {ext }}=1 \mathrm{~N} / \mathrm{m}^{2}$

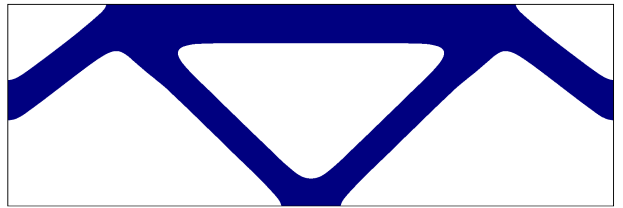

(b) $P_{\text {ext }}=1,250 \mathrm{~N} / \mathrm{m}^{2}$

Fig. 13 Optimal configurations for simple supported beam

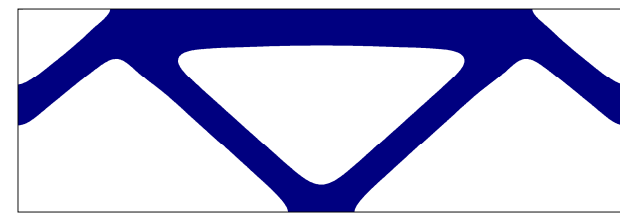

(a) $P_{e x t}=1 \mathrm{~N} / \mathrm{m}^{2}$

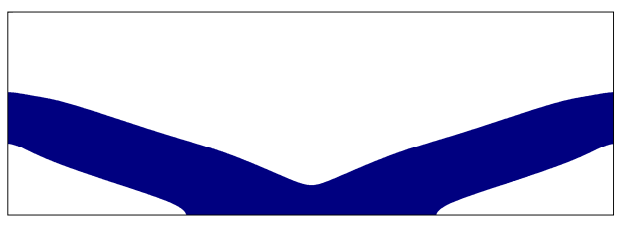

(b) $P_{\text {ext }}=1,250 \mathrm{~N} / \mathrm{m}^{2}$

Fig. 14 Optimal configurations for simple supported beam 
design loads when using linear finite element analysis. Figures 14(a) and (b) show optimal configurations when using the MPS method under different design loads, $P_{\text {ext }}=1 \mathrm{~N} / \mathrm{m}^{2}$ and $P_{\text {ext }}=1,250 \mathrm{~N} / \mathrm{m}^{2}$, respectively. The optimal configuration shown in Fig. 14(a) is the same as the result for the linear solution shown in Fig. 14(a) and Fig.13(a), since the deformation is adequately small. The optimal configuration in Fig. 14(b) shows no evidence of buckling under the set design load. These results indicate that the proposed method can obtain optimum and appropriate configurations for the set design loads.

\section{Conclusion}

This paper proposed a topology optimization method incorporating a level set boundary expression and a MPS method, and applied it to a minimum end compliance problem. We achieved the following:

(1) A strategy for integrating the topology optimization method incorporating the level set boundary expression with geometrically nonlinear analysis using the MPS method was developed.

(2) Based on the proposed topology optimization method, a minimum end compliance problem was formulated and an optimization algorithm considering the geometrical nonlinearity was then constructed.

(3) Several numerical examples were provided to confirm the usefulness of the proposed topology optimization method for minimum end compliance problems. We confirmed that smooth and clear optimal configurations considering geometrical nonlinearity were obtained when using the proposed topology optimization method. In addition, the obtained optimal configurations showed minimal dependency with respect to both initial configurations and particle size.

\section{References}

( 1 ) Bendsøe M.P. and Kikuchi N., Generating Optimal Topologies in Structural Design Using a Homogenization Method, Computer Methods in Applied Mechanics and Engineering, Vol.71, No.2, (1988), pp.197-224.

( 2 ) Matsui K., Terada K., Nishiwaki S. and Ishibashi Y., Consistent Topology Optimization Method for Structures with Finite Deformation, Transactions of Japan Society for Computational Engineering and Science, Vol.2001, (2001), p.20010046. (in Japanease)

( 3 ) Matsui K., Terada K. and Nishiwaki S., Topology Optimization with CAMD for Structures Undergoing Finite Deformation, IUTAM Symposium on Topology Design Optimization of Structures, Machines and Materials: Status and Perspectives, (2006).

( 4 ) Buhl T., Pedersen C.B.W. and Sigmund O., Stiffness Design of Geometrically Nonlinear Structures Using Topology Optimization, Structural and Multidisciplinary Optimization, Vol.19, No.2, (2000), pp.93-104.

( 5 ) Sekimoto T. and Noguchi H., Homologous Topology Optimization in Large Displacement and Buckling Problems, JSME International Journal, Series A, Vol.44, No.4, (2001), pp.616-622.

( 6 ) Bruns T.E. and Tortorelli D.A., Topology Optimization of Non-Linear Elastic Structures and Compliant Mechanisms., Computer Methods in Applied Mechanics and Engineering Vol.190, No.26-27, (2001), pp.3443-3459.

( 7 ) Bruns T.E., Sigmund O. and Tortorelli D.A., Numerical Methods for the Topology Optimization of Structures That Exhibit Snap-Through, International Journal for Numerical Methods in Engineering, Vol.55, No.10, (2002), pp.1215-1237.

( 8 ) Gea H.C. and Luo J., Topology Optimization of Structures with Geometrical Nonlinearities, Computers and Structures, Vol.79, No.20-21, (2001), pp.1977-1985.

(9) Cho S. and Jung H., Design Sensitivity Analysis and Topology Optimization of Displacement-Loaded Non-Linear Structures, Computer Methods in Applied Mechanics and Engineering, Vol.192, No.22-24, (2003), pp.2539-2553. 
(10) Jung D. and Gea H.C., Topology Optimization of Nonlinear Structures, Finite Elements in Analysis and Design, Vol.40, No.11, (2004), pp.1417-1427.

(11) Kemmler R., Lipka A. and Ramm E., Large Deformations and Stability in Topology Optimization, Structural and Multidisciplinary Optimization, Vol.30, No.5, (2005), pp.459-476.

(12) Yoon G.H. and Kim Y.Y., Element Connectivity Parameterization for Topology Optimization of Geometrically Nonlinear Structures, International Journal of Solids and Structures Vol.42, No.7, (2005), pp.1983-2009.

(13) Yoon G.H., Joung Y.S. and Kim Y.Y., Optimal Layout Design of Three-Dimensional Geometrically Non-Linear Structures Using the Element Connectivity Parameterization Method, International Journal for Numerical Methods in Engineering, Vol.69, No.6, (2007), pp.1278-1304.

(14) Belytschko T., Xiao S.P. and Parimi C., Topology Optimization with Implicit Functions and Regularization, International Journal for Numerical Methods in Engineering, Vol.57, No.8, (2003), pp.1177-1196.

(15) Wang M.Y., Wang X. and Guo D., A Level Set Method for Structural Topology Optimization, Computer Methods in Applied Mechanics and Engineering, Vol.192, No.(1-2), (2003), pp.227-246.

(16) Allaire G., Jouve F. and Toader A., Structural Optimization Using Sensitivity Analysis and a Level-Set Method, Journal of Computational Physics, Vol.194, No.1, (2004), pp.363-393.

(17) Kwak J. and Cho S., Topological Shape Optimization of Geometrically Nonlinear Structures Using Level Set Method, Computers and Structures, Vol.83, No.27, (2005), pp.2257-2268.

(18) Ha S.H. and Cho S., Level Set Based Topological Shape Optimization of Geometrically Nonlinear Structures Using Unstructured Mesh, Computers and Structures, Vol.86, No.(13-14), (2008), pp.1447-1455.

(19) Yamada T., Nishiwaki S., Izui K., Yoshimura M. and Takezawa A., A Structural Optimization Method Incorporating Level Set Boundary Expressions Based on The Concept of The Phase Field Method, Transactions of the Japan Society of Mechanical Engineers, Series A, Vol.48, No.753, (2009), pp.550-558. (in Japanease)

(20) Yamada, T., Izui, K., Nishiwaki, S. and Takezawa, A., A Topology Optimization Method Based on the Level Set Method Incorporating a Fictitious Interface Energy, Computer Methods in Applied Mechanics and Engineering, Vol.199, No.45-48, (2010), pp.28762891.

(21) Yamada, T., Izui, K. and Nishiwaki, S., A Level Set-Based Topology Optimization Method for Maximizing Thermal Diffusivity in Problems Including Design-Dependent Effects, Journal of Mechanical Design, Vol.133, No.3, (2011), p.031011.

(22) Belytschko T., Lu Y.Y. and Gu L., Element-Free Galerkin Methods, International Journal for Numerical Methods in Engineering, Vol.37, No.2, (1994), pp.229-256.

(23) Liu W.K., Jun S. and Zhang Y.F., Reproducing Kernel Particle Methods, International Journal for Numerical Methods in Fluids Vol.20, No.8-9, (1995), pp.1081-1106.

(24) Liu W.K., Jun S., Li S., Adee J. and Belytschko T., Reproducing Kernel Particle Methods for Structural Dynamics, International Journal for Numerical Methods in Engineering, Vol.38, No.10, (1995), pp.1655-1679.

(25) Belytschko T. and Black T., Elastic Crack Growth in Finite Elements with Minimal Remeshing, International Journal for Numerical Methods in Engineering, Vol.45, No.5, (1999), pp.601-620.

(26) Moës N., Dolbow J. and Belytschko T., A Finite Element Method for Crack Growth without Remeshing, International Journal for Numerical Methods in Engineering, Vol. 46, No.1, (1999), pp.131-150.

(27) Monaghan J.J., An Introduction to SPH, Computer Physics Communications, Vol.48, 
No.1, pp.89-96.

(28) Koshizuka S. and Oka Y., Moving Particle Semi-Implicit Method for Fragmentation of Incompressible Fluid, Nuclear Science and Engineering, Vol.123, No.3, (1996), pp.421434.

(29) Song M., Koshizuka S. and Oka Y., Dynamic Analysis of Elastic Solids by MPS method, Transactions of the Japan Society of Mechanical Engineers, Series A, Vol.71, No.701, (2005), pp.16-22. (in Japanease)

(30) Kondo M., Koshizuka S. and Suzuki Y., Application of Symplectic Scheme to ThreeDimensional Elastic Analysis Using MPS Method, Transactions of the Japan Society of Mechanical Engineers, Series A Vol.72, No.716, (2006), pp.425-431. (in Japanease)

(31) Inagaki K., Sakai M. and Koshizuka S., Response Analysis of a Concrete Cask Subjected to Seismic Motions by MPS Method, Transactions of Japan Society for Computational Engineering and Science Vol.2008, (2008), p.20080026. (in Japanease)

(32) Inagaki K., Sakai M. and Koshizuka S., Development of a Particle Method for Dynamic Elastic-Plastic Analysis, Transactions of Japan Society for Computational Engineering and Science Vol.2008, (2008), p.20080031. (in Japanease)

(33) Cho S. and Kwak J., Topology Design Optimization of Geometrically Non-Linear Structures Using Meshfree Method, Computer Methods in Applied Mechanics and Engineering, Vol.195, No.44-47, (2006), pp.5909-5925.

(34) Zhou J.X. and Zou W., Meshless Approximation Combined with Implicit Topology Description for Optimization of Continua, Structural and Multidisciplinary Optimization, Vol.36, No.4, (2008), pp.347-353. 